\title{
Epidemiología del traumatismo encéfalocraneano en niños del área suroriente de Santiago
}

\author{
Camilo Boza W. '; Alejandro Donoso $\mathrm{F}^{2}{ }^{2}$; Jorge Gigoux M. ${ }^{3}$; Adela Camus I. '; \\ Alejandro Bruhn C. ${ }^{2}$; Cristián Valverde G. ${ }^{2}$; Cristián Clavería R. ${ }^{2}$; Luis Villarroel del $\mathbf{P}^{3}$
}

\begin{abstract}
Resumen
Se estudiaron retrospectivamenle las caracteristicas epidemiológicos del traumatismo encéfalocraneono en 286 pacientes ingresados con ese diognóslico a un hospital general del Sevicio de Salud Melropolitano Sur-Oriente de Santiago de Chile, entre enero 1993 y abrit 1995 . Lc edad promedio de los pacientes era 6,1 años, $04,7 \%$ eran vorones, $54,5 \%$ escolares $(5$ a 14 años). $25,2 \%$ preescoiares ( 2 a menos de 5 añosi y $20,3 \%$ lactantes (menores de 24 meses]. En los loctantes y preescolares el accidente ocurrió con más frecuencia on el hogat [78,6\% y $60 \%$ respecliva mentel, en escolares en la vía púbica $(70,7 \%)$. En los primeros la principal causa del troumolismo fueron las caídas, en los escolores los accidentes de tránsito $(6,6 \%)$. En $64.9 \%$ de los casos la consulta fue hecho en la primera hora. El estado de la conciencia al ingresar al hospilol lescala de coma de Glasgowl. era 13 a 15 punkos en $76.9 \%$ de los pacientes, 9 a 12 puntos en 10,5\% de elios e igual o menor a 8 puntos en 10,8\%, proporciones que son mayores que las descritos en olros series.
\end{abstract}

(Palabros clare: iroumotismo encéfalocraneano.)

\section{Epidemiologic aspects of head injuries in children admitted to a public general hospital at Santiago, Chile}

Epidemiologic dalo on head injuries in children living at southeastern districts of mertopolitan Santiago. Chile, is described from a retrospective review of cinical records of 286 patients admitted to a university associated public general hospital from january 1993 throughour april 1995. Parients age (mean) al admission was 6.1 years, $64.7 \%$ patients were male, $54 \%$ schooi agec $15-14$ years!. $25.2 \%$ were children 2 to less than 5 years and $20.3 \%$ were intanls and todflers under 2 yeors. The most frequent sile of the accident in infants, toddlers and children under 5 vears wos their house $(78.6 \%$ and $60 \%$ cf coses respectively). while in school thildren it was the street $76.7 \%$. The ieading cause of head traumo in infants. roddlers and children under age 5 years were falls, while pedestrian injuries by molor vehicles predominated among school children $165.6 \%$ ). Sixly five percent of palients came to emergency rooms along the first hour trom the occident. According to the Glasgow Coma Scale score for consciousness at admission, $70.9 \%$ patients were classified as mildly affected iscores 13 to 15 l, $10.5 \%$ as moderately affected (scores 9 to 12 ) and $10.8 \%$ as severely altered [scores 8 or less'.

Koy words: head trauma .I

En los años más recientes el interés por el trauma ha aumentado en forma significativa. Los avances en el tratamiento médico y la salud

1. Internos Carrera de Medicina. Facultad de Medicina, Pontificia Universidad Católica de Chile.

2. Médicos, Unidad de Cuidados Intensivos Pediátricos, Hospital Dr. Sótero del Río. Servicio de Salud Merropolitano Sur-Oriente.

3. Unidad de Bioestadística. Centro Estudios Médicos, Facultad de Medicina Pontificia Universidad Católica de Chile. pública han tenido un gran impacto en la disminución de la mortalidad por otras causas, como las enfermedades infecciosas y respiratorias. No ha ocurrido lo mismo con los traumatismos que cada día asumen una mayor proporción de la mortalidad general.

En Estados Unidos los traumatimos son la principal causa de muerte en niños, y en particular el traumatismo encéfalocraneano (TEC) ${ }^{1-3}$. En Chile los traumatismos y envenenamientos son la tercera causa de muerte en toda la pobla- 
ción. El TEC es la primera dentro de ese grupo y, entre las edades de 5 y 14 años, el principal responsable de las muertes ${ }^{4}$, a pesar de lo cual hay poca información epidemiologica actualizada sobre el problema'. El objetivo de este trabajo fue describir el perfil epidemiológico del TEC en niños admitidos a un hospital general del área suroriente de Santiago metropolitano que atiende cerca de 170000 consultas de urgencia cada año.

\section{Pacientes y Método}

Se revisaron los registros de los 286 pacientes hospitalizados con el diagnóstico de TEC en el servicio de pediairia del Hospital Dr. Sótero del Río en un perfodo de 28 meses, entre enero de 1993 y abril de 1995. Se incluyeron en el análisis el sexo y edad del paciente: hora, día de la semana. lugar y mecanismo del accidente; tiempo transcumido hasta la consulta; unidad de ingreso; duración de la hospitalización y condición o estado al egresar

Los pacientes fueron ordenados según su edad en lactanses (menores de 2 años), preescolares ( 2 a menos de 5 anos) y escolates ( 5 a 14 años). La gravedad del traumatismo encefálico se calificó de acuerdo al puntaje de la escala de Glasgow para evaluación de la conciencias" asig. nado a cada caso al ingreso. clasificando luego a los pacientes como TEC grave si aquél era igual o menor que 8 ; moderudo entre 9 y 12 puntos y leve entre 13 y $15^{2}$.

La progresión del número de casos se comparó en los meses de enero y abril de cada año (1993, 1994 y 1995). La distribución mensual de casos en los ajos 1993 y 1994. Los dias en que ocurrieron los accidentes se ordenaron en: de semana (lunes a jueves), viernes, sábado, domingo, festivo y prefestivo.

Las comparaciones de proporciones se hicieron mediante prueba de Chi cuadrado o exacta de Fisher. Se considero significativo un valor $p<0,05$. Los datos fueron analizados con un programa estadístico SAS versión 6.01 para computadores personales compatibles.

\section{Resultados}

Durante el período estudiado se registraron 382588 consultas al servicio de urgencia; 1660 niños fueron atendidos y observados en la misma unidad a causa de traumatismo de cráneo y 286 fueron admitidos al servicio hospitalario de pediatría con el diagnóstico de traumatismo encéfalocraneano. La edad de estos 286 pacientes era $6,1 \pm 4,1$ años, $54,5 \%$ eran escolares, $25,2 \%$ preescolares, $20,3 \%$ lactantes y $64,7 \%$ eran varones. El número de casos admitidos en los meses de enero a abril de cada año aumentó en $135 \%$ (de 17 a 40 ) entre 1993 y 1994 , y $77 \%$ (de 40 a 71) entre los mismos meses de los años 1994 y 1995. Cuarenta y nueve por ciento de los TEC ocurrieron en el tercer trimestre, $27 \%$ en el primero y $24 \%$ en el segundo respectivamente. El mayor número de casos se registró en noviembre $(n=31)$. El número promedio admisiones por TEC fue de uno cada 2,5 días de semana y cada 3,5 días de fin de semana, viernes, fesivo o víspera de festivos ( $\mathrm{p}<0,005$ ), $55,9 \%$ de los accidentes ocurrieron entre las $16: 00$ y las $24: 00$ horas y $36,8 \%$ entre las $8: 00$ y las 16:00 horas. La mayoría de los accidentes causantes de TEC ocurrió en la vía pública $(54,1 \%)$, los restantes en el hogar $(40,6 \%)$ y ślo $3.6 \%$ sucedieron en establecimientos educacionales. La mayor parte de los TEC en lactantes y preescolares provino de accidentes en el hogar $(78,6$ y $60 \%$ respectivamente); en cambio en los escolares $76,7^{\circ}$ i ocurrió como consecuencia de accidentes en la vía pública. Las principales causas de TEC fueron las caídas $(46,2 \%), y$ los accidentes de tránsito $(45,9 \%)$. Del total de accidentes de tránsito el $73,1 \%$ correspondio a atropellos. Entre los lactantes el mecanismo más importante fueron las caídas menores $(50,0 \%)$, y en el grupo de preescolares, las caídas de altura $(31,0 \%)$. En los escolares, en cambio, el primer lugar lo ocuparon los accidentes de tránsito $(65,6 \%)$ (tabla). No se registraron TEC como consecuencia de prácticas recreacionales o deportivas.

El tiempo transcurrido entre accidente y consulta en el servicio de urgencia fue de 3,3 $\pm 10,2$ horas $(\overline{\mathrm{x}} \pm \mathrm{DE})$, siendo el mínimo 15 minutos y el máximo 72 horas, y $64,9 \%$ de los afectados consultaron dentro de la primera hora. De los pacientes cuyo TEC fue provocado por accidentes de tránsito, $84,3 \%$ consutaron durante la primera hora desde el suceso, lo que ocumió sólo en $51 \%$ de los afectados por accidentes en el hogar.

Un cuarto $(24,3 \%)$ de los casos ingresó a la unidad de cuidados intensivos (UCI), 41,2\% a la de cuidados intermedios y los restantes a sala. A pesar de no haber diferencias en la gravedad de los pacientes en los tres años de estudio, la proporción de los que fueron ingresados a cuidados intensivos o intermedios aumentó desde $42 \%$ en 1993 a $86 \%$ en 1995.La duración de la hospitalización fue de $5,9 \pm 8,8$ días. 
Tabla

Mecanismos de TEC en niños (\%)

\begin{tabular}{|c|c|c|c|c|c|}
\hline Mecanismbo & $\begin{array}{c}\text { Lactante } \\
(n=58)\end{array}$ & $\begin{array}{l}\text { Preescolar } \\
\quad(\mathrm{n}=71)\end{array}$ & $\begin{array}{l}\text { Escolar } \\
(n=154)\end{array}$ & $\begin{array}{c}\text { Total } \\
(n=283)\end{array}$ & Valor p \\
\hline Tránsito & 13,8 & 29.6 & 65.5 & 45,9 & $<0,0001^{*}$ \\
\hline atropello & 10,3 & 22.6 & 47.5 & 33,6 & $<0,0001^{*}$ \\
\hline $\begin{array}{l}\text { choque } \\
\text { caída vehículo }\end{array}$ & 3,4 & 4.2 & 3,2 & 3,5 & $<0,94$ \\
\hline en movimiento & 0.0 & 1.4 & 8.4 & 4,9 & $<0,012^{*}$ \\
\hline bicicletn & 0.0 & 1.4 & 6,5 & 3,9 & $<0,43^{*}$ \\
\hline Caidas & 77.6 & 64.8 & 26,0 & 46,3 & $<0,0001^{*}$ \\
\hline menores & 50,0 & 21.1 & 10.4 & 2.1 & $<0,0001^{*}$ \\
\hline altura & 15,5 & 31.0 & 11,7 & 17,3 & $<0,001^{* k}$ \\
\hline escalera & 12.0 & 12.7 & 3.9 & 7.8 & $<0,029^{*}$ \\
\hline Bicicleta & 0,0 & 2.8 & 4.5 & 3,2 & $<0,24$ \\
\hline Golpes & 6.9 & 1,4 & 3,9 & 3,9 & $<0,28$ \\
\hline Herida a bala & 0,0 & $\mathrm{t} .4$ & 0.0 & 0.4 & $<0,23$ \\
\hline Otros & 1.7 & 0.0 & 0.0 & 0.4 & $<0,15$ \\
\hline
\end{tabular}

* Diferencia estadísticamente significativa entre los grupos de edad. $p<0,05$.

En 220 (76.9\%) pacientes el puntaje de Glasgow de nivel de conciencia al ingresar era entre 13 y 15 , en otros $30(10,5 \%)$ de 9 a 12 y en 31 $(10,8 \%)$ era igual o menor que 8 . Entre los pacientes con Glasgow más bajo predominaban significativamente los escolares ( $p<0,03$ ). En $46,5 \%$ de los casos había fractura de cráneo. Se requirió tratamiento neuroquirúrgico en $7,7 \%$ de los niños hospitalizados por TEC.

El examen neurológico estaba alterado al alta en $7,3 \%$ de los afectados. Las alteraciones estaban representadas principalmente por paresias focales o déficit de pares craneanos. Cuatro pacientes fallecieron en este período a causa de hipertensión entracraneana refractaria a tratamiento: todos eran varones, escolares y en tres las lesiones habían sido causadas por accidentes de tránsito.

En $15,7 \%$ de los pacientes se registró otro diagnóstico asociado, el más frecuente fue politraumatismo $(6,6 \%)$. En todos las lesiones eran consecuencias de accidentes en la vía pública. En tres pacientes hubo evidencias de subluxación entre vértebras cervicales. En 5,8\% de los escolares afectados se encontraron antecedentes de sindrome de déficit atencional. En siete casos había razones para sospechar fuertemente de maltrato infantil; en uno de ellos se confirmo abuso sexual.

\section{Comentario}

Estos resultados concuerdan por lo general con los de otros informes ${ }^{8}$ en que el TEC de los niños afecta predominantemente a los varones y tiene entre las principales causas las caídas y los accidentes de tránsito. Diferencias de localización geográfica y estrato socioeconómico pueden explicar que en algunas experiencias predominen, como en este caso, los accidentes en la vía pública o en el hogar ${ }^{5}, 9$. En nuestro ambien- 
$\therefore$ los establecimientos educacionales parecen sr bastante seguros pues en ellos ocurrieron luy pocos de los accidentes registrados. Otro echo relevante fue la ausencia de casos de EC debidos a prácticas deportivas, salvo alguos accidentes en bicicleta, a diferencia de 10 escrito en otros países, donde causan hasta $1 \%$ de dicha lesiones ${ }^{8}$.

El aumento del número de hospitalizaciones n los tres años del estudio podria ser atribuido I sostenido aumento del número de accidentes , también, a mayor tendencia a considerar nezsaria la vigilancia directa de estos pacientes. uesto que no se encontró mayor incidencia de 'EC durante los fines de semana, festivos, vieres y prefestivos, sino más bien en los días háiles, es posible suponer que el desplazamiento acia y desde los centros educacionales repreinta un riesgo para esta comunidad en particuIr. La mayor incidencia d accidentes con TEC n primavera, es concordante con la de otros iformes ${ }^{8}$.

Los accidentes en la vía pública no sólo son nportantes por su incidencia y por la frecuenia en que figuran como causa de TEC, sino ambién porque las lesiones que producen incuen en mayor morbilidad y letalidad ${ }^{3}$, y por ser ssceptibles de prevenir con campañas de conol de tránsito y educación de conductores, scolares y padres. Como en otras series las priveras causas de TEC en lactantes y preescolass fueron las caídas y ocurrieron en el hogar, ımbién susceptibles de evitar con medidas simles de educación, control y protección contra esgos en el hogar del afectado ${ }^{10.11}$. El maltrato ebe considerarse como una posibilidad cuando I mecanismo del trauma parece poco preciso. fecta a lactantes o existen otras lesiones asoiadas como fracturas de extremidades o hemoagia retinal, por ejemplo ${ }^{12-14}$.

El tiempo transcurrido entre el accidente y la onsulta fue bastante adecuado en esta serie ${ }^{5}$. a proporción de pacientes con calificaciones e Glasgow igual o menor a 8, así como las de lectados por fracturas de cráneo y alteraciones n la tomografía axial cerebral, fueron mayores $n$ ésta que en otras series, sugieren mayor graedad de las lesiones, que podría explicarse en arte por mecanismos causales de mayor enería y porque una proporción importante de paientes con traumatismos de cráneo (supuesta- mente leves), fueron dados de aita después de algunas horas de observacion en el servicio de urgencias ${ }^{5,1} 15$.

La naturaleza prospectiva de este análisis no permitió describir la evolución de los pacientes con alteraciones neurológicas al alta ni el eventual desarrollo de trastornos conductuales y cognitivos entre los aparentemente sanos al egresar ${ }^{16}$.

Es evidente que el TEC como problema de salud no puede ser resuelto en el hospital. Mientras los accidentes de tránsito aumentan año a año en Chile, en otros países las muertes por accidentes de Iránsito y TEC han disminuido gracias a políticas de prevención y sistemas eficientes de rescate y transporte de las víctimas ${ }^{17}$. Por otra parte los padres juegan un rol fundamental en la educación, control y aplicación de medios y medidas de seguridad para sus hijos en el hogar.

\section{Referencias}

1. Anoinino: Division of Injury Control, Center for Environmental Health and Injury Control, Centers for Disease Control. Childhood injuries in the United States. Am J Dis Child 1990: 144: 627-646.

2. Mayer $T$. Walker $M$, Johnson $D$, et al: Causes of morbidity and mortality in severe pediatric trauma. JAMA 1981: 245: 719-721.

3. Rivara FP: Epidemiology and prevention of pediatric brain injury. Pediatr Ann 1994; $23: 12-17$.

4. Anónimo: Anuario Demograftico de] [nstituto Nacional de Estadisticas. 1993: 318-336.

5. Arriagada $S$, Cerda $M$, Abarca $S$, Muñoz $G$, Torres $R$, Corrales R: Traumatismo encéfalocraneano en nit̄os. Rev Chil Pediatr 1995; 66: 196-199

6. Jennes B. Teasdale $G$ : Assesment of coma and impaired conciousness. A practical scale. Lancet 1974; I]: 81-84,

7. Genuarelli TA: Initial assessment and management of head injury. In: Pits LH, Wagner FK Jr., Eds. Craniospinal truma. New York: Thieme Medical 1990: 11 . 24.

8. Kraus $J F$, Rock A, Hemyari P: Brain injuries among infants, children, adolescents and young adults. Am J Dis Child 1990; 144: 684-691.

9. Kraus JF, Fife D, Ramstein $K$, Conroy $C$, Cox P: The relationship of family income to the incidence, causes, and outcome of serious brain injury, San Diego County, California. Am J Public Health 1986; 76: 1345-1347.

10. Chadwick $D L$, Chin $S$, Salerno C, Landsverk $J$. Kitchen $L$ : Deaths from falls in children: how far is fatal?. J Tramma 1991: 31 : 1353-1355. 
11. Williams RA: Injuries in infants and small children resulring from witnessed and corroborated free falls. J Trauma 1991: 31 : 1350-1352.

12. Burkos'ski HM: Neurological injury in child abuse. Presented at the congress of Neurological Surgeons Annual Meeting: ost 31-nov 5, 1992; Washington DC.

13. Duhatme AC. Alario AJ, Lewander J, ef at: Head injury in very young children: mechanisms, injury types. and ophthalnologic findings in 100 hospitalized patients younger than two years of age. Pedialrics 1992; 90 : $179-185$.
14. Hardwood-Nash DC: Abuse to the pediatric central nervous system. American Journal of Neuroradiology. $1992 ; 13 ; 569-575$.

15. Kraus JF. Fife D. Cox $P$. Ramstein $K$, Conroy $C$ : Incidence, severity, and external causes of pediatric brain injury. Am J Dis Child 1986; 140: 687-693.

16. Jaffe XM. Fay GC. Polisarr NL. et al: Severity of pediatric traumatic brain injury and neurobehavioral recovery at I year-a cohort study. Arch Phys Med Rehabil 1993: 74: 587-595.

17. Hockelnan RA: A pediatricians view: causes of death among young. Pediatr Ann 1992; $21: 407-408$.

\section{AVISO A LOS AUTORES}

Por acuerdo del Comité Editorial, la Revista Chilena de Pediatría devolverá sin tramitar todos los trabajos que no den estricto cumplimiento al Reglamento de Publicaciones y a las Instrucciones de los Autores que se editan en cada número de la Revista. 